\title{
Contrast Media: Safety Issues and ESUR Guidelines
}

\author{
H.S. Thomsen and J.A.W. Webb, eds.
}

Berlin, Germany: Springer-Verlag, 2009, 254 pages, $\$ 149$

This second edition of Contrast Media: Safety Issues and ESUR Guidelines provides new and updated information on the contrast-related safety issues that a radiologist can encounter in daily practice. Comprehensive information is presented on potential adverse reactions from the use of iodine-based contrast media, MRI contrast agents, ultrasound contrast agents, and barium.

The book is aimed at radiologists, radiology residents, and fellows. Other nonradiologists and their trainees, such as neurologists, neurosurgeons, orthopedists, cardiologists, and radiation therapists who have imaging facilities and use contrast media, can also benefit from the information, as can allied health professionals working with radiologists and nonradiologists.

The book is organized into several sections (30 chapters) that present comprehensive information on general issues regarding contrast media and their adverse reactions. Also, specific clinical situations and the use of contrast media are described. The guidelines of the European Society of Urogenital Radiology (ESUR) are stated at the end of the book.

Section 1 is 6 chapters covering general issues, including classification and terminology, requests for contrastenhanced imaging, the legal aspects of off-label use of medicines, pharmacovigilance, the approval process for new contrast agents, and adverse events after contrast agent use.

Section 2 addresses iodinated and gadolinium contrast agents and is divided into 3 subsections. The first subsection, on general adverse reactions, contains 2 chapters on the prevention and management of acute adverse reactions. The second subsection, on renal adverse effects, has 3 chapters describing contrast medium-induced nephropathy and the use of contrast media on dialysis patients and noninsulin-dependent diabetic patients. Four chapters on other adverse reactions comprise the last subsection. These chapters deal with pregnancy and lactation, pheochromocytoma, interactions with other drugs, and extravasation injury.
Section 3 covers iodinated contrast media and has 4 chapters describing late adverse reactions and the effects of contrast media on blood, endothelium, the lungs, and thyroid function.

Section 4, on MRI contrast media, has 3 subsections. The first subsection ( 3 chapters) deals with non-tissue-specific extracellular media and describe chelates and stability, diagnostic efficacy, and radiography with gadolinium contrast agents. The second subsection ( 2 chapters) presents gadolinium-based contrast agents and discusses acute and delayed adverse reactions, including nephrogenic systemic sclerosis. The last subsection (2 chapters) describes gadolinium-based and non-gadolinium-based contrast agents.

Section 5 presents the safety of ultrasound contrast media, and Section 6 deals with safety issues concerning the use of barium preparations.

The book concludes with an appendix that cites the ESUR guidelines on contrast media and the official publications from the ESUR.

This is an excellent book that I highly recommend. It belongs in the libraries of radiologists and training programs. This comprehensive review is written in such a way that the flow of information is preserved throughout the book. The multiple authors and editors are to be commended for such dedication and detail. Day-to-day imaging and management scenarios encountered by radiologists are easily found in this resource book.

The reader is advised to compare the American College of Radiology guidelines on contrast media, which can be found online, with those in this book, given the differences in the availability and use of contrast media between Europe and the United States.

Aurelio Matamoros, Jr.

M.D. Anderson Cancer Center

Houston, Texas 